\title{
Transferability of Parameters of Strictly Local Geminals' Wave Function and Possibility of Sequential Derivation of Molecular Mechanics*
}

\author{
A. M. TOKMACHEV ${ }^{1 \dagger}$ A. L. TCHOUGRÉEFF ${ }^{2}$ \\ ${ }^{1}$ Institute of Inorganic Chemistry, RWTH Aachen, Prof.-Pirlet-Str. 1, 52056 Aachen, Germany \\ ${ }^{2}$ Karpov Institute of Physical Chemistry, Vorontsovo pole 10, 105064 Moscow, Russia \\ Received 19 August 2004; Accepted 7 December 2004 \\ DOI 10.1002/jcc.20188 \\ Published online in Wiley InterScience (www.interscience.wiley.com).
}

\begin{abstract}
The problem of substantiation of molecular mechanics (MM) remains actual due to growing popularity of hybrid quantum/classical (QM/MM) schemes. Recently proposed deductive molecular mechanics (DMM) seems to be a natural tool to derive mechanistic models of molecular energy (classical force fields) from a suitable quantum mechanical (QM) description of molecular structure. It is based on an assumption that the trial wave function underlying the MM description is one of the antisymmetrized product of strictly local geminals (SLG). A proof of transferability of electronic structure parameters (ESPs) in this approximation is an essential component of a logical framework for the transition from the QM to an MM description because it allows constructing expressions for potential energy surfaces by proper consideration of the response of the ESPs to the variations of geometry parameters. In the present article the ESPs defining density matrix elements and basis one-electron states (hybrid orbitals-HOs) in the SLG approximation are formally considered. The transferability of the density matrix elements with respect to the parameters of molecular electronic structure and the linear response relations for the HOs are proven to take place under very nonrestrictive conditions. Special attention is paid to numerical estimates of the ESPs' features giving an "experimental" support to approximate expressions for the molecular energy.
\end{abstract}

(C) 2005 Wiley Periodicals, Inc. J Comput Chem 26: 491-505, 2005

Key words: molecular mechanics (MM); quantum/classical schemes

\section{Introduction}

Molecular mechanics $(\mathrm{MM})^{1,2}$ is currently a versatile and very popular tool in laboratory and industrial practice. It remains the most practical way of analyzing potential energy surfaces (PES) for large molecules even though linear scaling $O(N)$ methods of quantum chemistry are becoming available. ${ }^{3,4}$ The nature of the MM as a combination of totally empirical classical force fields allows to realize its main drawbacks (e.g., inapplicability to highly correlated systems) and advantages (fast evaluation of the total energy and its gradient and high accuracy of molecular geometries obtained) and to characterize it as the most preferable according to the "quality/cost" criterion but with the field of application limited to a certain combination of properties, processes, and classes of molecules.

Despite its long history and a wealth of successful applications, the MM approach remains not substantiated theoretically. Substantiation of MM is not merely an academic question. The last years have demonstrated a growing interest ${ }^{5-8}$ to hybrid quantum mechanical/molecular mechanical (QM/MM) schemes where differ- ent parts of the entire molecule are treated by different methods. Although the QM/MM methods shift the limits for numerical applications, they require more insight into the electronic structure underlying the MM schemes because it is not clear how to construct the junction between quantically and classically treated subsystems. Although many ad hoc recipes for the junction construction are proposed in the literature (see, e.g., reviews ${ }^{6-8}$ ), the problem has not been yet solved, which leads to numerous artifacts in QM/MM schemes reviewed in ref. 8. The general formal solution of this problem proposed in refs. 9 and 10 is based on the assumption that a generic MM scheme can be obtained from a

* Dedicated to Prof. N. F. Stepanov on the occasion of his 70th birthday.

${ }^{\dagger}$ AvH Postdoctoral Fellow, on leave from the Karpov Institute of Physical Chemistry, Moscow, Russia.

Correspondence to: A. L. Tchougréeff; A. M. Tokmachev; e-mail: andrei@ cc.nifhi.ac.ru

Contract/grant sponsor: RFBR; contract/grant numbers: 02-03-32087, 04-03-32146, and 04-03-32206 
suitable QM method by a series of approximations. Thus, the prospect of development of effective QM/MM schemes constitutes the practical aspect of the general task of the MM substantiation. An important prerequisite for the announced derivation of MM from QM is the choice of the quantum chemical method underlying the MM description. By taking a proper form of the trial wave function having some common features (or resemblance) with the MM schemes we can significantly simplify the task. The representation of the total energy of the covalently bound molecule in the current MM schemes is tightly bound to the concept of twocenter bonds. The energy is taken as a sum of intrabond, bending, torsion, and nonbonding contributions. The specific forms of force fields depend on the implementation, and quite a lot of schemes with systems of parameters fitted to reproduce various characteristics of different classes of molecules have been proposed. ${ }^{12-18}$ In the course of the evolvement of the MM approach increasingly sophisticated contributions are added to the original simplistic picture, ${ }^{2}$ which allow extension of the MM approach to more and more complex and not transparently tractable classes of the molecules. ${ }^{19-21}$ Thus, an acceptable substantiation of the MM should not be reduced to deriving or validating any specific MM scheme. By contrast, a generic mechanistic picture must be obtained on the basis of the adequate QM description.

The standard QM methods in that or another manner based on the delocalized picture of the molecular electronic structure provided by the SCF approximation are not quite suitable for the purpose of the MM substantiation. First, the general SCF energy expression contains contributions of the same form for all pairs of atoms, and separation of the energy into bonding and nonbonding contributions is not built into the SCF methods. Second, even applying different localization techniques intended to reconcile the SCF picture of the electronic structure with the chemist's view relying upon the bond's concept leads not to very much progress due to "tails" of the localized orbitals, which are difficult for handling and absolutely nontransferable (it is also impossible to prove theoretically the transferability of the MO LCAO coefficients or, even, of the SCF density matrix elements).

The above discussion allows specification of some criteria for selecting a QM method suitable to serve as a starting point for the MM derivation: discrimination and different treatment of bonding and nonbonding contributions to the energy, strict locality of one-electron states, variational determination of the electronic structure parameters (ESPs). Failure to fulfill any of these criteria leads to a necessity to take whichever of the mentioned features as assumptions (as, e.g., in ref. 22, where the analysis was based on the PCILO method ${ }^{23}$ ).

The unability of the SCF approximation to be a basis for the additivity concept in general (and thus for the MM in particular) was clear even on very early stage of the theory development. Yet then (see ref. 24) it had been proposed to use a geminal-based description for substantiating the additive methods and the bond energy concept. However, the one-electron carrier spaces to be used for geminal construction had not been sufficiently specified, and the entire geminal-based scheme had not been explored numerically to a due extent. Recently we proposed ${ }^{25,26}$ a semiempirical method that satisfies the suitability criteria formulated above. It uses the trial wave function in the form of antisymmetrized product of strictly local geminals (SLG), and variationally determines the strictly local one-electron states and the density matrix elements. Numerical implementations with the MINDO/ $3^{25,27}$ and $\mathrm{NDDO}^{26}$ parameterizations of the semiempirical Hamiltonian have shown that the SLG method supercedes the SCF one in the description of the heats of formation, molecular geometries, and ionization potentials of organic molecules. Incidentally, it provides a good basis for description of molecular electronic structure in chemical terms. The approximate transferability of the ESPs of the SLG approach was numerically demonstrated in ref. 28, where it was shown that the energy calculated with some ESPs "fixed" and characteristic for a particular class of atoms and for bonds is close to that obtained by direct minimization of the SLG energy. More refined treatment was proposed in refs. 29 and 30, where linear response relations for the form of one-electron states were used to derive explicit expressions for the angular dependence of the energy in the case of $s p^{3}$ hybridized carbon and nitrogen atoms. The numerical estimates of the MM force field parameters obtained in ref. 29 on the basis of the analytical expressions for the constants of force fields are close to those accepted in the MM. Special attention was paid to off-diagonal force fields and to possible sources of the angular dependence of the energy ${ }^{29}$ as well as those of pyramidalization potential in nitrogen-containing compounds. ${ }^{30}$ The development of these ideas has led to the formulation of the "deductive molecular mechanics," 31 which represents atoms by their hybridization tetrahedra (see below) of different shapes adjusted according to geometry and valence state variations rather than by harmonically interacting point masses ("balls and springs") used in standard MM. All these results are obtained within the assumptions of (1) the perfect transferability of ESPs characterizing chemical bonds, and (2) of the validity of linear response relations for hybridization with respect to geometry variations. In the present article we address the problem of transferability of the density-related ESPs, explore the precision and the validity limits for the linear response formulae for the shapes of hybridization tetrahedra, and consider the possibility of recovering the standard MM description from the deductive molecular mechanics.

This article is organized as follows: in Section 2 we briefly review the main features of the SLG method (with the MINDO/3 semiempirical parameterization) relevant to constructing the MM description. In Section 3.1 the ESPs characterizing the density distribution in chemical bonds and lone pairs as they appear in the SLG method are analyzed and the features assuring their transferability are singled out. In Section 3.2 we briefly discuss the structure of the hybridization manifold and give expressions for the variations of the hybridization-related ESPs in response to geometry changes. Results of numerical experiments are given in these sections to support our theoretical derivation of transferability and of linear response relations. In Section 4.1 we discuss different possible approximate descriptions of the ESPs and energy leading to various versions of the deductive MM theory. In Section 4.2 we consider the possibility of deriving the standard MM picture from the DMM by projecting out excessive (from the MM point of view) variables of the latter and give theoretical expressions for the force fields' parameters of the standard MM. Finally, we discuss the relation between the transferability of the density related ESPs and that of the MM force fields. 


\section{SLG Description of Molecular Electronic Structure}

Constructing the SLG trial wave function according to refs. 25 and 26 requires the following moves. First, the one-electron basis of the strictly local hybrid orbitals (HOs) must be constructed. These orbitals are obtained by an orthogonal transformation of the $s$ and $p$ AOs for each "heavy" (nonhydrogen) atom. These transformations are represented by $4 \times 4$ orthogonal matrices $h^{A} \in O(4)$ for each heavy atom $A$. All the HOs are assigned either to respective two-electron chemical bonds or to electron lone pairs. Each chemical bond refers to two such HOs: $|r\rangle$ and $|l\rangle$ (right- and left-end ones, respectively). Each lone pair is formed by one HO only (a right one for the sake of definiteness).

Chemical bonds and lone pairs are described by singlet twoelectron functions- geminals ${ }^{32}$ taken in the form originally proposed by Weinbaum. ${ }^{33}$ With use of the second quantization notation they are written as:

$$
g_{m}^{+}=u_{m} r_{m \alpha}^{+} r_{m \beta}^{+}+v_{m} l_{m \alpha}^{+} l_{m \beta}^{+}+w_{m}\left(r_{m \alpha}^{+} l_{m \beta}^{+}+l_{m \alpha}^{+} r_{m \beta}^{+}\right)
$$

for chemical bonds and:

$$
g_{m}^{+}=r_{m \alpha}^{+} r_{m \beta}^{+}
$$

for lone pairs. The normalization condition imposed on the amplitudes reads:

$$
u_{m}^{2}+v_{m}^{2}+2 w_{m}^{2}=1
$$

The geminals thus defined in the carrier space spanned by so constructed HOs are termed to be strictly local geminals (SLG).

The wave function of electrons in the molecule is then taken as the antisymmetrized product of the geminals given by eqs. (1) and (2):

$$
|\Phi\rangle=\prod_{m} g_{m}^{+}|0\rangle
$$

The SLG energy is a function of the intrabond matrix elements of spinless one- and two-electron density matrices:

$$
\begin{gathered}
P_{m}^{t t^{\prime}}=\left\langle 0\left|g_{m} t_{m \sigma}^{+} t_{m \sigma}^{\prime} g_{m}^{+}\right| 0\right\rangle, \Gamma_{m}^{t t^{\prime}}=\left\langle 0\left|g_{m} t_{m \beta}^{+} t_{m \alpha}^{\prime+} t_{m \alpha}^{\prime} t_{m \beta} g_{m}^{+}\right| 0\right\rangle, \\
P_{m}^{r r}=u_{m}^{2}+w_{m}^{2}, P_{m}^{l l}=v_{m}^{2}+w_{m}^{2}, P_{m}^{r l}=P_{m}^{l r}=\left(u_{m}+v_{m}\right) w_{m}, \\
\Gamma_{m}^{r r}=u_{m}^{2}, \Gamma_{m}^{l l}=v_{m}^{2}, \Gamma_{m}^{r l}=\Gamma_{m}^{l r}=w_{m}^{2},
\end{gathered}
$$

where $t$ and $t^{\prime}(= \pm 1)$ correspond to the right $(+1)$ and to the left $(-1)$ ends of the bond and to the Fermi operators $r$ and $l$, respectively. Certain energy contributions further reduce to Coulomb interaction of effective atomic charges residing on the atoms. Applying the Mulliken scheme for the charges to the SLG wave function yields them in the form:

$$
Q_{A}=2 \sum_{t_{m} \in A} P_{m}^{t t}-Z_{A}
$$

If the Hamiltonian of the MINDO/3 form ${ }^{34}$ in the $\mathrm{HO}$ basis is used with the SLG trial wave function the total energy can be written in a form somewhat close to the MM energy with interactions between bonded and nonbonded atoms treated in different ways and closely relates it to that given in ref. 22 in the context of analysis of a variety of additive schemes of molecular energy:

$$
\begin{gathered}
E=\sum_{A} E_{A}+\sum_{A<B} E_{A B}, \\
E_{A}=\sum_{t_{m} \in A}\left[2 U_{m}^{t} P_{m}^{t t}+\left(t_{m} t_{m} \mid t_{m} t_{m}\right)^{T_{m}} \Gamma_{m}^{t t}\right]+2 \sum_{\substack{t_{k} k_{h}^{\prime} \in A \\
k<m}} g_{t_{k} t_{m}}^{T_{k}} P_{k}^{t t} P_{m}^{t^{\prime} t^{\prime}}, \\
E_{R_{m} L_{m}}^{b o n d}=2 \gamma_{R_{m} L_{m}}\left[\Gamma_{m}^{r l}-2 P_{m}^{r r} P_{m}^{l l}\right]-4 \beta_{r_{m} l_{m}}^{R_{m} L_{m}} P_{m}^{r l}, \\
E_{A B}^{n o n b o n d}=Q_{A} Q_{B} \gamma_{A B}+Z_{A} Z_{B} D_{A B},
\end{gathered}
$$

where $T_{m}$ refers to the atom bearing the $\mathrm{HO} t_{m} ; D_{A B}$ is MINDO/3 specific core-core repulsion; the reduced Coulomb integral is:

$$
g_{t_{k} t_{m}^{\prime}}^{T_{k}}=2\left(t_{k} t_{k} \mid t_{m}^{\prime} t_{m}^{\prime}\right)^{T_{k}}-\left(t_{k} t_{m}^{\prime} \mid t_{m}^{\prime} t_{k}\right)^{T_{k}}
$$

and the resonance (electron hopping) one is:

$$
\beta_{t_{m_{1}} t_{m_{2}}}^{A B}=\sum_{i \in A} \sum_{j \in B} h_{m_{1} i}^{A} h_{m_{2} j}^{B} \beta_{i j}^{A B}
$$

In this energy expression it is assumed that the nonbonding contribution appears for all pairs of atoms, whereas the bonding is only for those with an actual bond.

Molecular integrals entering the above expressions depend on molecular geometry and on the orthogonal matrices $h^{A}$ for all nonhydrogen atoms $A$. The expressions for the matrix elements in the $\mathrm{HO}$ basis are given in ref. 25 .

The energy expression eq. (7) depends on the amplitudes of bond geminals through the values of $P_{m}^{t t^{\prime}}$ and $\Gamma_{m}^{t t^{\prime}}$, and on the form of HOs through the molecular integrals. The amplitudes of two ionic $\left(u_{m}, v_{m}\right)$ and one covalent (Heitler-London type, $\left.\sqrt{2} w_{m}\right)$ configurations in eq. (1) for each geminal are determined with the use of the variational principle as well as the matrices $h^{A}$ of transformation of the $\mathrm{AO}$ basis to the $\mathrm{HO}$ one.

This comprises the essence of the semiempirical SLG method.

\section{Electronic Structure Parameters in the SLG Picture}

In the previous section we briefly reviewed the semiempirical implementation of the SLG method for analysis of the electronic structure and expressed the total molecular energy in the form of eq. (7), which allows the representation of the molecular PES as a sum of local increments. These increments depend on the ESPs of two classes: (1) those defining the hybridization of atomic basis 
sets, and (2) the intrabond density matrix elements. In this section we concentrate upon the proof of transferability of the electron density matrix elements, related to the geminal amplitudes, and on the structure of the hybridization manifold as it appears in the SLG approximation.

\section{Density Matrix Elements and Their Transferability}

As is reported in Section 2, the energy in the SLG approximation is a function of one- and two-electron density matrices. Their matrix elements are, in turn, expressed through the geminal amplitudes, appearing while diagonalizing the effective bond Hamiltonians. Thus, any analysis of the properties of the density ESPs starts from description of the latter.

\section{Effective Bond Hamiltonians}

Within the original SLG approach ${ }^{25,26}$ the geminals are characterized by the amplitudes [see eq. (1)] $u_{m}, v_{m}$, and $z_{m}=\sqrt{2} w_{m}$ [which simplifies the normalization condition eq. (3) for the amplitudes to: $\left.u_{m}^{2}+v_{m}^{2}+z_{m}^{2}=1\right]$. To determine them, the effective Hamiltonians for each bond geminal are constructed. The optimal values of these amplitudes are the solution of the eigenvector problem (see also ref. 35 ):

$$
\left(\begin{array}{ccc}
A_{m} & D_{m} & 0 \\
D_{m} & C_{m} & D_{m} \\
0 & D_{m} & B_{m}
\end{array}\right)\left(\begin{array}{c}
u_{m} \\
z_{m} \\
v_{m}
\end{array}\right)=\varepsilon^{m}\left(\begin{array}{c}
u_{m} \\
z_{m} \\
v_{m}
\end{array}\right)
$$

corresponding to its lowest eigenvalue.

The matrix elements of the effective bond Hamiltonians are defined as (with the MINDO/3 Hamiltonian):

$$
\begin{aligned}
& A_{m}=2 U_{m}^{r}+\left(r_{m} r_{m} \mid r_{m} r_{m}\right)^{R_{m}}- 4 \gamma_{R_{m} L_{m}} P_{m}^{l l} \\
&+2 \sum_{B \neq R_{m}} \gamma_{R_{m} B} Q_{B}+2 \sum_{\substack{t_{m_{1}} \in R_{m} \\
m_{1} \neq m}} g_{r_{m} t_{m_{1}}}^{R_{m}} P_{m_{1}}^{t t} ; \\
& B_{m}=2 U_{m}^{l}+\left(l_{m} l_{m} \mid l_{m} l_{m}\right)^{L_{m}}-4 \gamma_{R_{m} L_{m}} P_{m}^{r r} \\
&+2 \sum_{B \neq L_{m}} \gamma_{L_{m} B} Q_{B}+2 \sum_{\substack{t_{m} \in L_{m} \\
m_{1} \neq m}} g_{l_{m} t_{m_{1}}}^{L_{m}} P_{m_{1}}^{t t} ; \\
& C_{m}=\frac{1}{2}\left(A_{m}+B_{m}\right)-\Delta \gamma_{m} ; D_{m}=-\sqrt{2} \beta_{r_{m} l_{m}}^{R_{m} L_{m}},
\end{aligned}
$$

where

$$
\Delta \gamma_{m}=g_{m}-\gamma_{R_{m} L_{m}}, g_{m}=\frac{1}{2} \sum_{t \in\{r, l\}}\left(t_{m} t_{m} \mid t_{m} t_{m}\right)^{T_{m}}
$$

The calculations of refs. 25 and 26 performed on organic compounds of different classes (alkanes, alcohols, amines, etc.) have demonstrated a remarkable stability of all the geminal-related ESPs. The values of the polarity $P_{m}^{r r}-P_{m}^{l l}$ do not exceed 0.07 by absolute value for the compounds containing carbon, nitrogen, and hydrogen atoms (for the situation with oxygen and fluorine, see below). Also, the ionicity $u_{m}^{2}+v_{m}^{2}$ for a rich variety of bonds has a stable value about 0.4 . The bond orders $2 P_{m}^{r l}$ all acquire values between 0.92 and 1.0. These features, although not completely unexpected because the transferability of the parameters of the single bonds in organic compounds is well known experimentally, require a theoretical explanation.

\section{Pseudospin Representation and the Perturbative Estimates of the Bond-Geminal ESPs}

To provide the required explanation we notice that the effective Hamiltonians for the bond geminals can be represented as a sum of the unperturbed part, which, when diagonalized, yields an invariant, that is, exactly transferable, values of the ESPs and of a perturbation responsible for specificity of different chemical compositions and environments.

Pseudospin Operator of the Bond Geminal. Let us introduce a pseudospin $\hat{\tau}_{m}$ operator corresponding to the pseudospin value $\tau_{m}=1$. The matrices of its components in the basis of the configurations defining the geminal are given by:

$$
\begin{gathered}
\hat{\tau}_{z m}=\left(\begin{array}{ccc}
1 & 0 & 0 \\
0 & 0 & 0 \\
0 & 0 & -1
\end{array}\right), \hat{\tau}_{+m}=\left(\begin{array}{ccc}
0 & \sqrt{2} & 0 \\
0 & 0 & \sqrt{2} \\
0 & 0 & 0
\end{array}\right), \\
\hat{\tau}_{-m}=\left(\hat{\tau}_{+m}\right)^{\dagger} .
\end{gathered}
$$

The configurations with $\left\langle\hat{\tau}_{z m}\right\rangle= \pm 1$ are the ionic ones with both electrons located on the same end of chemical bond (right or left, respectively). In terms of the pseudospin operator averages, the quantities in eq. (5) are expressed as follows:

$$
\begin{gathered}
P_{m}^{t t}=\frac{1}{2}\left(1+t\left\langle\hat{\tau}_{z m}\right\rangle\right), P_{m}^{r l}=\frac{1}{2}\left\langle\hat{\tau}_{+m}\right\rangle, P_{m}^{l r}=\frac{1}{2}\left\langle\hat{\tau}_{-m}\right\rangle, \\
\Gamma_{m}^{t t}=\frac{1}{2}\left(\left\langle\hat{\tau}_{z m}^{2}\right\rangle+t\left\langle\hat{\tau}_{z m}\right\rangle\right), \Gamma_{m}^{r l}=\Gamma_{m}^{l r}=\frac{1}{2}\left(1-\left\langle\hat{\tau}_{z m}^{2}\right\rangle\right) .
\end{gathered}
$$

The effective bond Hamiltonians can be rewritten in terms of the pseudospin operators introduced just above. Indeed, the effective Hamiltonian eq. (10) for each of the bond-representing geminals has the form:

$$
\begin{gathered}
H_{m}^{e f f}=H_{0 m}^{e f f}+\Delta_{I m} \hat{\tau}_{z m}^{2}+\Delta_{P m} \hat{\tau}_{z m}, \\
H_{0 m}^{e f f}=\left(\begin{array}{ccc}
C_{m} & D_{m} & 0 \\
D_{m} & C_{m} & D_{m} \\
0 & D_{m} & C_{m}
\end{array}\right), \\
\Delta_{I m}=\frac{1}{2}\left(A_{m}+B_{m}\right)-C_{m}=\Delta \gamma_{m}, \Delta_{P m}=\frac{1}{2}\left(A_{m}-B_{m}\right),
\end{gathered}
$$

where the part of the effective Hamiltonian proportional to $\hat{\tau}_{z m}^{2}$ is responsible for reproducing the relative contribution of the ionic and covalent configurations to the bond geminal, and the contribution proportional to $\hat{\tau}_{z m}$ relates to the asymmetry (polarity) of the bond. The geminal amplitudes obtained by diagonalizing the unperturbed bond Hamiltonians $H_{0 m}^{\text {eff }}$ and the density or $\hat{\tau}$-type ESPs thus obtained are perfectly invariant: 


$$
\begin{gathered}
u_{0 m}=v_{0 m}=w_{0 m}=\frac{1}{2},\left(z_{0 m}=\frac{1}{\sqrt{2}}\right), P_{0 m}^{t t^{\prime}}=\frac{1}{2}, \Gamma_{0 m}^{t t^{\prime}}=\frac{1}{4}, \\
\left\langle\hat{\tau}_{z m}\right\rangle_{0}=0,\left\langle\hat{\tau}_{z m}^{2}\right\rangle_{0}=\frac{1}{2},\left\langle\hat{\tau}_{+m}\right\rangle_{0}=\left\langle\hat{\tau}_{-m}\right\rangle_{0}=1
\end{gathered}
$$

with respect to the sorts of atoms and to the geometry parameters, although the unperturbed effective Hamiltonians $H_{\mathrm{Om}}^{\text {eff }}$ themselves are, of course, not. The invariant values of the ESPs are also rather close to the exact SLG values obtained in refs. 25 and 26.

\section{Perturbative Estimate of ESPs with Respect to Noncorrelated Bare} Hamiltonian. One can try to estimate the optimal values of the ESPs specific for each bond and molecule perturbatively by using the linear response approximation. ${ }^{36}$ According to the latter, the response $\delta\langle A\rangle$ of a quantity described by the operator $A$ to the perturbation $\lambda B$ of the Hamiltonian (where $\lambda$ is the parameter characterizing the intensity of the perturbation) has the form:

$$
\delta\langle A\rangle=\lambda\langle\langle A ; B\rangle\rangle
$$

where the zero frequency Green's function in the energy domain is given by the relation:

$$
\langle\langle A ; B\rangle\rangle=2 \sum_{i \neq 0}\langle 0|A| i\rangle\left(\varepsilon_{0}-\varepsilon_{i}\right)^{-1}\langle i|B| 0\rangle .
$$

Inserting the amplitudes of the geminals for the ground and the two excited states of the bond and two excitation energies (which are equal to $\sqrt{2}\left|D_{m}\right|$ and $2 \sqrt{2}\left|D_{m}\right|$ ) for the effective Hamiltonian $H_{\mathrm{O} m}^{\text {eff }}$ to the general expression for the Green's functions eq. (18) one can immediately check that only the diagonal (with respect to $A$ and $B$ running over the relevant components of the pseudospin operator and its squared $z$-component) Green's functions are nonvanishing. Therefore, the first-order responses of the averages of the pseudospin operator components can be written as:

$$
\begin{gathered}
\left\langle\hat{\tau}_{z m}\right\rangle=\delta\left\langle\hat{\tau}_{z m}\right\rangle=\frac{B_{m}-A_{m}}{4 \beta_{r_{m} l_{m}}^{R_{m} L_{m}}}, \\
\delta\left\langle\hat{\tau}_{z m}^{2}\right\rangle=-\frac{\Delta \gamma_{m}}{8 \beta_{r_{m} l_{m}}^{R_{m} L_{m}}}, \\
\delta\left\langle\hat{\tau}_{+m}+\hat{\tau}_{-m}\right\rangle=0 .
\end{gathered}
$$

The last expression demonstrates that at least in the linear response approximation the bond order does not change (i.e., is invariant even for the different atoms forming the bond and geometries). This result, though suggests certain stability of the bond orders, is in fact a consequence of the SCF type of the two-electron wave function implicitly assumed by the decomposition of the bond effective Hamiltonian eq. (15). The unperturbed part thus defined yields the symmetric ground state with the total weight of the ionic configurations equal to that of the covalent one. This coincides with the result characteristic for the SCF approximate treatment of the symmetric bond. It is obvious that the formulae for the bond- order variation and the two-electron density matrix elements (and the SCF approximation itself) are not valid at larger interatomic separations [the denominators in eq. (19) become too small] although the exact solution of the SLG problem has a correct asymptotic behavior for single bonds even at infinite interatomic separations. This attractive feature of the SLG model is lost in the perturbative treatment based on the Hamiltonian separation eq. (15) as it is in the SCF approximation as well.

Perturbation of the Density Matrix Elements for Correlated Ground State. To overcome the above failure of the perturbative estimation of the two-electron density and of the bond orders, let us consider a symmetric bond. This would correspond to a different decomposition of the effective bond Hamiltonian than that of eq. (15). We assume that the contribution to the effective bond Hamiltonian, which is proportional to $\hat{\tau}_{z m}^{2}$ is included into the unperturbed (zero order) Hamiltonian. The problem then reduces to a $2 \times 2$ matrix diagonalization. The ESPs, as they appear from solution of this problem, are:

$$
\Gamma_{m}^{t t^{\prime}}=\frac{1}{4}\left(1-t t^{\prime} \frac{1}{\Gamma\left(\zeta_{m}\right)}\right), P_{m}^{t t}=\frac{1}{2}, P_{m}^{r l}=\frac{\zeta_{m}}{2 \Gamma\left(\zeta_{m}\right)},
$$

where

$$
\zeta_{m}=4 \beta_{r_{m} l_{m}}^{R_{m} L_{m}} / \Delta \gamma_{m}, \Gamma\left(\zeta_{m}\right)=\sqrt{1+\zeta_{m}^{2}}
$$

Small interatomic separations characteristic for the real bonds correspond to the limit $\zeta_{m} \gg 1$ and the ESPs have the asymptotic behavior as:

$$
\Gamma_{0 m}^{t t^{\prime}}=\frac{1}{4}\left(1-t t^{\prime} \frac{1}{\zeta_{m}}\right), P_{m}^{r l}=\frac{1}{2}\left(1-\frac{1}{2 \zeta_{m}^{2}}\right)
$$

Now, when the total ionic contribution to the geminal is calculated exactly (variationally), the bond polarity can be estimated perturbatively in the linear response approximation, but with the correlated ground state of the symmetricly effective bond Hamiltonian taken for evaluating the Green's function. It can be conveniently done with use of a dimensionless asymmetry parameter:

$$
\mu_{m}=\frac{B_{m}-A_{m}}{\Delta \gamma_{m} \Gamma\left(\zeta_{m}\right)}
$$

Inserting the corresponding ground state to the definition of the Green's function $\left\langle\left\langle\hat{\tau}_{z m} ; \hat{\tau}_{z m}\right\rangle\right\rangle$ one obtains:

$$
\left\langle\hat{\tau}_{z m}\right\rangle=\mu_{m} \frac{\Gamma\left(\zeta_{m}\right)-1}{\Gamma\left(\zeta_{m}\right)+1}
$$

for the polarity of the bond between atoms with the fixed hybridizations. It vanishes for infinite interatomic separation as it should be for the exact wave function. The bond ionicity and the bond order are not affected in the linear response approximation.

The expression for the bond polarity coincides with that of eq. (24) even if the second-order perturbation correction to the wave 
function is used (i.e., the contribution to the bond polarity proportional to $\mu_{m}^{2}$ is absent). At the same time the second-order corrected bond ionicity and bond order have the following form:

$$
\begin{gathered}
\left\langle\hat{\tau}_{z m}^{2}\right\rangle=\left\langle\hat{\tau}_{z m}^{2}\right\rangle_{c}\left[1+\mu_{m}^{2} \frac{2 \Gamma\left(\zeta_{m}\right)+1}{2\left(\Gamma\left(\zeta_{m}\right)+1\right)}\right], \\
\left\langle\hat{\tau}_{+m}+\hat{\tau}_{-m}\right\rangle=\left\langle\hat{\tau}_{+m}+\hat{\tau}_{-m}\right\rangle_{c}\left[1+\mu_{m}^{2} \frac{2 \Gamma\left(\zeta_{m}\right)+1-\Gamma^{2}\left(\zeta_{m}\right)}{2\left(\Gamma\left(\zeta_{m}\right)+1\right)^{2}}\right],
\end{gathered}
$$

where the ESPs with the subscript $c$ correspond to the estimates by eq. (20).

\section{Lone Pairs}

Another archetypical form of two-electron group is the lone pair. As is mentioned above, the lone pair is described by a degenerate geminal containing the contribution of only one ionic configuration. For the sake of definiteness we set it to be the right-end ionic configuration of the corresponding degenerate bond [the amplitude $u_{m}$ becomes equal to unity, see eq. (2)]. The ESPs related to the lone pair can be readily evaluated:

$$
\begin{gathered}
\left\langle\hat{\tau}_{z m}\right\rangle=\delta\left\langle\hat{\tau}_{z m}\right\rangle=1, \delta\left\langle\hat{\tau}_{z m}^{2}\right\rangle=\frac{1}{2}, \\
P_{m}^{r l}=P_{m}^{l r}=0, \Gamma_{m}^{r r}=1, \Gamma_{m}^{l l}=\Gamma_{m}^{r l}=\Gamma_{m}^{l r}=0 .
\end{gathered}
$$

These quantities are perfectly invariant and transferable from one molecule to another and basically characterize (within the accepted approximation, of course) the qualitative difference between the atoms of different chemical elements: by the number of lone pairs they bear.

\section{Numerical Estimates of Density ESPs' Transferability}

The above analytical results must be controlled by numerical estimates to get a feeling of the real sense of the "first" and "second" orders. Table 1 represents the results of calculations on the ESPs $\left\langle\hat{\tau}_{z m}\right\rangle,\left\langle\hat{\tau}_{z m}^{2}\right\rangle$, and $\left\langle\hat{\tau}_{+m}\right\rangle$ by the SLG method [eq. (10)] and by the approximate formulae [eqs. (19), (20), (22), (24), and (25)] for some characteristic bonds in small molecules. The results show that in the case of bonds with small polarity all the formulae perform very well. The most precise approximations [eqs. (24) and (25)] give results that perfectly coincide with the exact (SLGMINDO/3) ones even for very polar $\mathrm{O}-\mathrm{H}$ and $\mathrm{F}-\mathrm{H}$ bonds. Also, estimates according to the asymptotic $\left(\zeta_{m} \gg 1\right)$ formulae [eq. (20)] give reasonable results for the ESPs of the bonds in not too polar molecules at their equilibrium geometries. The main source of stability of the bond order values is the validity of the above limit, which in its turn, takes place due to the fact that the difference between one- and two-center electron-electron repulsion integrals $\left(\Delta \gamma_{m}\right)$ at interatomic separations characteristic for chemical bonding is much smaller than the resonance interaction at the same distance. The data of Table 1 illustrate the difference between that which may be called MM atom types. For example, the primary $\mathrm{C}-\mathrm{H}$ bonds in the ethane and propane molecules have very similar ESPs, at the same time somewhat differing from those for the secondary $\mathrm{C}-\mathrm{H}$ bonds in the propane molecule.

Further analysis of the quantities $\mu_{m}$ allows to single out two types of factors loaded upon this parameter: those related to the bond itself (which are again hybridization dependent) and the rest describing the environment of the bond. These factors contribute additively:

$$
\mu_{m}=\mu_{0 m}+\mu_{1 m}
$$

where the intrinsic bond-related part is:

$$
\begin{array}{r}
\mu_{0 m}=\frac{1}{\Delta \gamma_{m} \Gamma\left(\zeta_{m}\right)}\left[2\left(U_{m}^{l}-U_{m}^{r}\right)+\left(l_{m} l_{m} \mid l_{m} l_{m}\right)^{L_{m}}-\left(r_{m} r_{m} \mid r_{m} r_{m}\right)^{R_{m}}\right. \\
\left.\quad+\sum_{\substack{t_{m} \in L_{m} \\
m_{1} \neq m}} g_{l_{m} t_{m_{1}}}^{L_{m}} n_{m_{1}}-\sum_{\substack{t_{m} \in R_{m} \\
m_{1} \neq m}} g_{r_{m} t_{m_{1}}}^{R_{m}} n_{m_{1}}\right], \quad \text { (28) }
\end{array}
$$

where $n_{m}$ equals 1 for an incident chemical bond and 2 for a lone pair. This contribution is characteristic for the pair of atoms $R_{m} L_{m}$ with given ratios of the $s$ - and $p$-weights in the HOs $\left|r_{m}\right\rangle$ and $\left|l_{m}\right\rangle$ ascribed to the bond at hand and clearly depending on the chemical nature of these atoms through specific values of atomic parameters and numbers of lone pairs.

The contribution to the bond asymmetry coming from the environment of the bond is:

$$
\begin{gathered}
\mu_{1 m}=\frac{1}{\Delta \gamma_{m} \Gamma\left(\zeta_{m}\right)}\left[2 \sum_{B \neq L_{m}} \gamma_{L_{m} B} Q_{B}-2 \sum_{B \neq R_{m}} \gamma_{R_{m} B} Q_{B}+4 \gamma_{R_{m} L_{m}}\left\langle\hat{\tau}_{z m}\right\rangle\right. \\
\left.+2 \sum_{\substack{t_{m_{1}} \in L_{m} \\
m_{1} \neq m}} g_{l_{m} t_{m_{1}}}^{L_{m}} t_{m_{1}}\left\langle\hat{\tau}_{z m_{1}}\right\rangle-2 \sum_{\substack{t_{m_{1}} \in R_{m} \\
m_{1} \neq m}} g_{r_{m} t_{m 1}}^{R_{m_{m}}} t_{m_{1}}\left\langle\hat{\tau}_{z m_{1}}\right\rangle\right] .
\end{gathered}
$$

In the molecules where all bonds are weakly polar one can expect that the external part $\mu_{1 m}$ is small. Also, in the molecules containing many polar bonds, the effect of randomly distributed effective atomic charges almost vanishes by this leading to small values of external contributions $\mu_{1 m}$. Thus, the only situation when one could expect the environment to affect the characteristics of the otherwise transferable bond is that when the bond under consideration falls to a close vicinity with a few strongly charged atoms arranged in such a way that their fields sum up to a nonzero overall field acting along the bond. That is, clearly, one of the situations that elaborated MM parameterizations mark as a special one, requiring specific values of parameters. The separation of $\mu_{m}$ according to eq. (27) allows obtaining transferable, environmentindependent approximating functions for the ESPs by substituting into eqs. (24) and (25) the parameter $\mu_{0 m}$ instead of $\mu_{m}$. The numerical results are given in Table 1 . It shows that this approach leads to the approximate ESPs perfectly coinciding with those obtained by the SLG method itself that demonstrates the applicability of such a scheme. The comparison of data in Table 1 allows singling out the effects of the environment. For example, the primary $\mathrm{C}-\mathrm{H}$ bonds in the ethane and propane molecules result in 


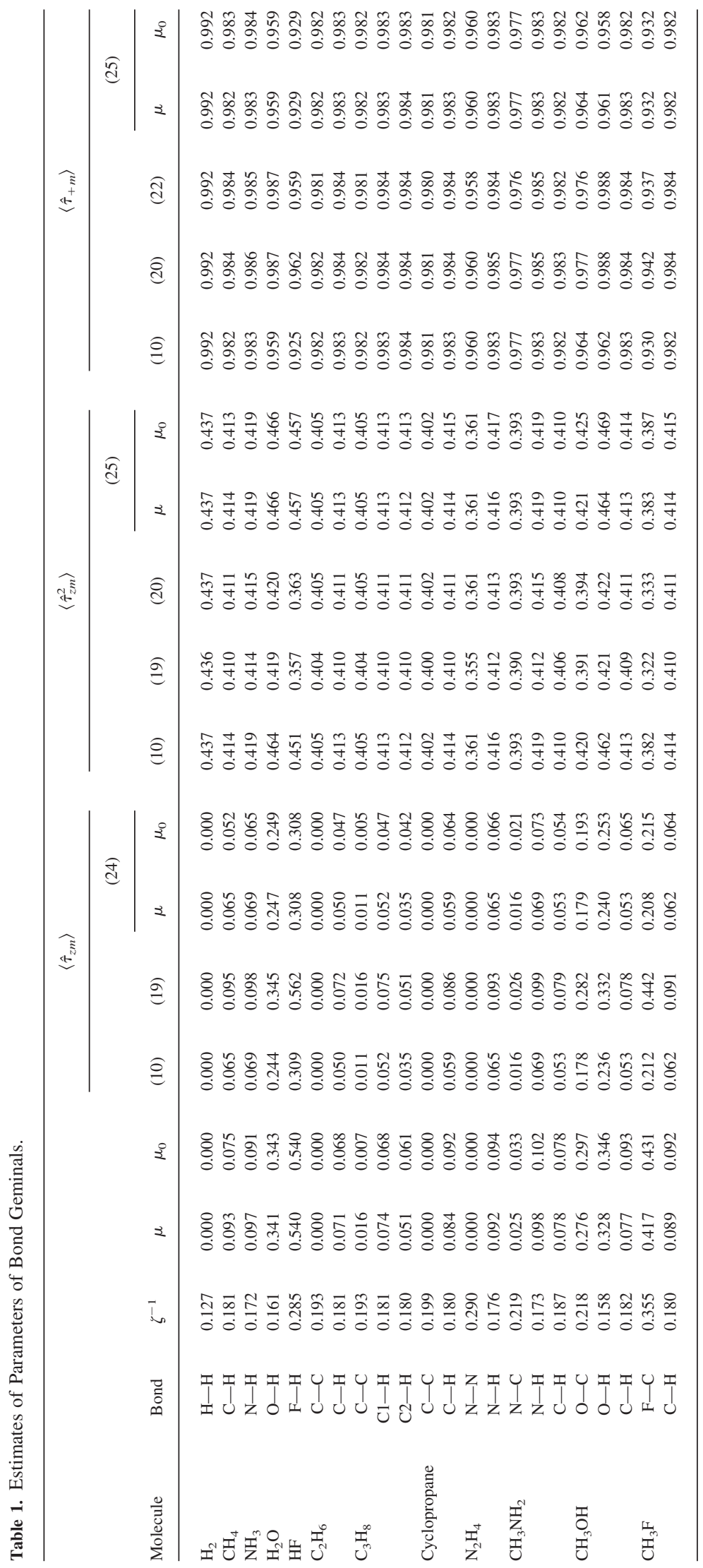


coinciding values of the ESPs estimated by using $\mu_{0}$. Therefore, the small difference between the ESPs of these bonds obtained by the SLG method is totally caused by the slightly different environment, that is, by the $\mu_{1}$ values, which are equal to 0.003 and 0.006 , respectively. The small magnitude of the deviations between the SLG and approximately estimated ESPs can be rationalized by the smallness of their effect on the total energy of the molecule. For example, even in the case of a polar water molecule the approximation of the bond ESPs by their values based on the $\mu_{0}$ estimates leads to an increase of the total energy by only 0.014 $\mathrm{kcal} / \mathrm{mol}$ compared to the exact SLG calculation.

\section{Mathematical Description of Hybridization in the SLG Picture}

In the framework of the SLG scheme the structure of one-electron basis states is defined by orthogonal transformations of AOs for each atom with an $s p$-valence shell. The energy expression eq. (7) is the function of the parameters defining these transformations. The $4 \times 4 O(4)$ matrix $h^{A}$ of transformation from the AO to the HO basis set on the atom $A$ depends on six angular variables. Three of them (pseudorotation angles $\vec{\omega}_{b}=\left(\omega_{s x}, \omega_{s y}, \omega_{s z}\right)$ with subscripts indicating pairs of basis AOs mixed by the corresponding $2 \times 2$ Jacobi rotations) define the structure of the HOs ( $s$-/ $p$-mixing and relative directions of the HOs) while other three [quasirotation angles $\vec{\omega}_{l}=\left(\omega_{y z},-\omega_{x z}, \omega_{z y}\right)$ ] define the $S O(3)$ matrix performing rotation of the set of four HOs as a whole (prefix quasi refers to the fact that no physical body rotates under its action, only the system of HOs). Generally, the transformation of orbitals caused by a pseudorotation forms a set of HOs that is known as hybridization pattern (like $s p^{3}, s p^{2}$, etc.), which is more or less stable, while the set of quasirotation angles is totally nontransferable, depends on the relative placement of bonded atoms and, obviously, is governed by the resonance contribution to the energy because only the latter depends on the directions of the HOs.

The mathematical description of hybridization is based on employing the algebraic group structure of the hybrids' manifold. Due to the latter, any small variation of HOs in a vicinity of a given set of HOs represented by a $4 \times 4$ orthogonal matrix $h$ can be expressed with use of the $S O(4)$ matrix $H$ close to the unity matrix:

$$
\begin{gathered}
H=I+\delta^{(1)} H+\delta^{(2)} H, \\
h^{\prime}=H h \approx h+\delta^{(1)} h+\delta^{(2)} h, \\
\delta^{(1)} h=\delta^{(1)} H h, \delta^{(2)} h=\delta^{(2)} H h .
\end{gathered}
$$

The general form of matrix $H$ is analyzed in ref. 31, where its expansion up to the second order in the vicinity of the unity matrix is given. Also, simple expressions for the first-order variation of the structure of the HOs due to small quasi- and pseudorotations $\delta \vec{\omega}_{l}$ and $\delta \vec{\omega}_{b}$ applied to the set of HOs at a given atom are derived:

$$
\delta^{(1)} s=-\left(\delta \vec{\omega}_{b}, \vec{v}\right),
$$

$$
\delta^{(1)} \vec{v}=s \delta \vec{\omega}_{b}+\delta \vec{\omega}_{l} \times \vec{v}
$$

where $\times$ stands for the vector product of 3 -vectors and we use a quaternion $(s, \vec{v})$ representation (see ref. 31 and below) of the HOs where the coefficient $s$ at the $s$-AO in the HO LCAO expansion is a scalar and the coefficients at the $p$-AOs form the vector part $\vec{v}$ of the quaternion. Because we know the dependence of the molecular integrals (and, therefore, energy) on the HO LCAO coefficients we find according to eq. (31) their dependence on the ESPs $\vec{\omega}_{b}$ and $\vec{\omega}_{l}$ defining hybridization.

\section{Deductive Molecular Mechanics and Possibility of Derivation of Standard MM}

\section{Basics of Deductive Molecular Mechanics}

The analysis of the properties of the ESPs pertinent to the SLG approximation performed above allows rewriting the energy [eq. (7)] as follows:

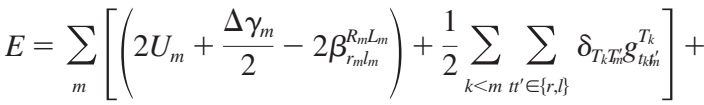

$$
\begin{aligned}
& +\sum_{A<B} Z_{A} Z_{B} D_{A B}+ \\
& +\sum_{m} \sum_{t \in\{r, l\}}\left[t\left\langle\hat{\tau}_{z m}\right\rangle\left(U_{m}^{t}+\frac{1}{2}\left(t_{m} t_{m} \mid t_{m} t_{m}\right)^{T_{m}}\right)+\right. \\
& +\frac{1}{2} \sum_{k<m} \sum_{t t^{\prime} \in\{r, l\}} \delta_{T_{k} T_{m}^{\prime}} g_{t_{k} t^{\prime}}^{T_{k}}\left(t\left\langle\hat{\tau}_{z k}\right\rangle+t^{\prime}\left\langle\hat{\tau}_{z m}\right\rangle\right)+ \\
& +\sum_{m}\left[\sum_{t \in\{r, l\}} \frac{1}{2}\left(t_{m} t_{m} \mid t_{m} t_{m}\right)^{T_{m}}-\gamma_{R_{m} L_{m}}\right] \delta\left\langle\hat{\tau}_{z m}^{2}\right\rangle+ \\
& +\sum_{A<B} Q_{A} Q_{B} \gamma_{A B}+\sum_{m} \gamma_{R_{m} L_{m}}\left\langle\hat{\tau}_{z m}\right\rangle^{2}+ \\
& +\frac{1}{2} \sum_{k<m} \sum_{t t^{\prime} \in\{r, l\}} \delta_{T_{k} T_{m}} g_{t_{k} k h^{\prime}}^{T_{k}} t t^{\prime}\left\langle\hat{\tau}_{z k}\right\rangle\left\langle\hat{\tau}_{z m}\right\rangle+ \\
& -\sum_{m} \beta_{r_{m} l_{m}}^{R_{m} L_{m}} \delta\left\langle\hat{\tau}_{+m}+\hat{\tau}_{-m}\right\rangle
\end{aligned}
$$

This representation allows the following family of approximate treatments for the energy. If the geminal amplitude-related ESPs are fixed at their transferable values-the corresponding approximation is called the FA, that is, the fixed amplitudes one-the energy [eq. (32)] reduces to the lines (a) and (b), which yields an expression dependent on the molecular geometry and the hybridization ESPs only. The other lines in eq. (32) reappear if corrections to the amplitude-related ESPs are taken into account. This corresponds to the amplitude tuning in response to the geometry variations or environment details, and this family of approxima- 
tions is thus called the TA, that is, the tuned amplitudes approximation. The simplest one in this family is that which retains only the terms linear in $\left\langle\hat{\tau}_{z m}\right\rangle \sim \mu_{m}$ [lines (c) and (d)], thus allowing the bond asymmetry (polar bonds and nonvanishing effective charges). Including the terms linear in $\zeta_{m}^{-1}$ provides the possibility to take into account the variations of two-electron density matrices in response to geometry and environment variations [line (e)], but only including the quadratic terms yields corrections to the bond orders [line (h)]. Further, in this article we mean by the TA approximation its simplest ( $\mu_{m}$-linear) version. Because the corrections to the bond orders appear only in the second order in small parameters $\mu_{m}$ and $\zeta_{m}^{-1}$ the FA and the simplest TA approximations may have some value as can be seen from Table 1 by comparing columns (10), (19), and (20).

Further components of the description are those related to the HOs. The latter enter into the theory through the Hamiltonian matrix elements in the $\mathrm{HO}$ basis. The matrix elements entering eqs. (7) and (32) are either invariant with respect to basis transformations (the interatomic Coulomb interaction $\gamma_{A B}$ ), or can be uniquely expressed through contributions of $s$-AO to the HOs (the one-center matrix elements). The only class of molecular integrals depending on the whole structure of the HOs (including directions) is that of the resonance integrals. As we mentioned in Section 3.2, each $s p^{x}$-HO can be considered as a normalized quaternion $(s, \vec{v})$. Following ref. 31 we represent the entire system of HOs at any given atom by four vector parts $\vec{v}_{m}$ of the corresponding orthonormal quaternions. Even this representation is superfluous, because only six Jacobi angles suffice to describe the system of HOs of each given atom completely. Nevertheless, usage of the vector parts is visual. If the latter are assumed to have the corresponding nucleus as their common origin, the tetrahedral shape thus obtained contains (with an excess) all necessary information about the system of HOs of the given atom. In ref. 31 such a construct was called the hybridization tetrahedron of the heavy atom at hand. Using the hybridization tetrahedra as elements of the theoretical construct allows further discrimination of possible approximations. Due to the mentioned dependencies of the molecular integrals on the Jacobi angles both the FA and TA approximations to the energy [eq. (32)] depend on the relative orientation of the hybridization tetrahedra through the bond resonance integrals $\beta_{r_{m} l_{m}}^{R_{m} L_{m}}$ The resonance integrals also depend on the shapes (relative weights of the $s$ - and $p$-contributions to the HOs, which ultimately define the interhybrid angles) of the hybridization tetrahedra. All other terms in eq. (32) in the FA and TA approximations depend only on the shapes of the hybridization tetrahedra. This leads to the possibility to either fix the relative weights of the $s$ - and $p$-orbitals (FO, i.e., fixed orbitals approximation) at $s p^{n}(n=1 \div 3)$ or any other allowable values, and by this fix the shapes of the hybridization tetrahedra, which thus become interacting rigid bodies or to allow the relative weights of the $s$ - and p-orbitals to be tuned, thus leading to the TO_tuned orbitals - picture of the flexible hybridization tetrahedra. Whichever combination of the FA or TA treatments for the density matrix elements, on the one hand, with the FO or TO treatments for the HOs, on the other hand, results in a representation of the molecular energy [eq. (32)] as such of the system of tetrahedral bodies (rigid or flexible) whose interactions and self-energies depend on distances between their centers, their shapes, and relative orientations. For example, the energy variation due to small pseudo- and quasirotations of the hybridization tetrahedron in the vicinity of the equilibrium for $s p^{3}$-hybridized carbon atom in the FA approximation is given by a diagonal quadratic form:

$$
\begin{array}{r}
\delta_{\omega \omega}^{(2)} E=2\left(\left(\delta \vec{\omega}_{b}^{R_{m}}\left|\varphi_{b b}^{R_{m} R_{m}}\right| \delta \vec{\omega}_{b}^{R_{m}}\right)+\left(\delta \vec{\omega}_{l}^{R_{m}}\left|\mathscr{G}_{l l}^{R_{m} R_{m}}\right| \delta \vec{\omega}_{l}^{R_{m}}\right)\right), \\
\mathcal{G}_{l l}^{R_{m} R_{m}}=\frac{4}{\sqrt{3}}\left(\beta_{\zeta \sigma}^{R_{m} L_{m}} S_{m}^{L_{m}}-\beta_{\zeta \zeta}^{R_{m} L_{m}} \sqrt{1-\left(s_{m}^{\left.L_{m}\right)^{2}}\right)} \mathscr{I},\right. \\
\mathcal{G}_{b b}^{R_{m} R_{m}}=2\left[\left(\beta_{\sigma \sigma}^{R_{m} L_{m}}+\frac{1}{\sqrt{3}} \beta_{\zeta \sigma}^{R_{m} L_{m}}\right) s_{m}^{L_{m}}\right. \\
\left.-\left(\beta_{\sigma \zeta}^{R_{m} L_{m}}+\frac{1}{\sqrt{3}} \beta_{\zeta \zeta}^{R_{m} L_{m}}\right) \sqrt{1-\left(s_{m}^{L_{m}}\right)^{2}}\right] I,
\end{array}
$$

Three equal eigenvalues (diagonal elements) correspond to variations of $\vec{\omega}_{b}$, while three other equal eigenvalues correspond to those of $\vec{\omega}_{l}$. Following the general reasoning, ${ }^{37}$ this can be treated as a mechanistic model for molecular energy, for which the name "deductive molecular mechanics" has been coined in ref. 31 . These constructs apparently satisfy the conditions formulated in the Introduction for a QM-derived mechanistic model of molecular PES. However, there are several questions that need to be clarified. First, we have to see what might be the relation between the DMM and the standard MM known in the literature. Second, the precision of the FA, TA, FO, and TO approximate treatments must be evaluated. Third, the interrelation between the ESPs and the molecular geometry must be established. The rest of the article is devoted to investigating these three topics.

\section{Relation between Deductive and Standard MM}

\section{General Setting}

The content of the deductive molecular mechanics as formulated in ref. 31 , and above is a description of the molecular energy in the form of eq. (32) as a function of shapes and mutual orientations of the hybridization tetrahedra and of geometry parameters. On the other hand, the standard MM can be qualified as a scheme directly parameterizing the molecular energy as a function of molecular geometry only. From this point of view the Jacobi angles variables $\vec{\omega}_{b}, \vec{\omega}_{l}$, describing the shapes and orientations of hybridization tetrahedra, are superfluous and must be excluded. This can be done by finding the response of the corresponding ESPs to the variations of bond lengths and valence angles with use of linear response relations between different subsets of variables pertinent to the DMM picture. To do so let us consider a minimum of the energy with respect to both geometry and the ESPs. In the vicinity of a minimum the energy can be expanded up to second-order with respect to nuclear displacements $q$ and variations of the ESPs $x$ :

$$
\begin{aligned}
E= & E_{0}+\frac{1}{2}\left(x-x_{0}\left|\nabla_{x} \nabla_{x} E\right| x-x_{0}\right)+\left(x-x_{0}\left|\nabla_{x} \nabla_{q} E\right| q-q_{0}\right) \\
& +\left(q-q_{0}\left|\nabla_{q} \nabla_{x} E\right| x-x_{0}\right)+\frac{1}{2}\left(q-q_{0}\left|\nabla_{q} \nabla_{q} E\right| q-q_{0}\right),
\end{aligned}
$$


where linear terms disappear due to minimum conditions. For the sake of definiteness we restrict ourselves by the FA picture. Then the only remaining ESPs are the Jacobi angles describing the shapes and orientations of the hybridization tetrahedra. Minimization of the energy [eq. (34)] with respect to $x$ for a given value of $q$ leads to basic linear response relation between the ESPs and the geometry distortions:

$$
x-x_{0}=-\left(\nabla_{x} \nabla_{x} E\right)^{-1} \nabla_{x} \nabla_{q} E\left(q-q_{0}\right) .
$$

The scheme of this type was used in ref. 38 to demonstrate the transferability of scaling factors typically applied to reach an agreement between the calculated ( $a b$ initio) IR frequencies and the experimental ones.

\section{Linear Response Relations for Hybridization ESPs}

The main use of formulae (35) is for exclusion of the angular variables describing the hybridization tetrahedra from the mechanistic picture. Now we estimate the precision of the linear response relations [eq. (35)] between geometry and hybridization variations themselves by numerical study of elongation of one $\mathrm{C}-\mathrm{H}$ bond and deformations of valence angles. We consider the tetrahedral methane molecule as a reference [its parameters then correspond to subscript 0 in eqs. (34) and (35)]. First of all, we notice that the $\nabla_{x} \nabla_{x} E$ matrix further simplifies for methane since $s_{m}^{L_{m}}=1$ and, therefore, simple analytical expressions become possible. Also, we remark that the FA approximation is adequate here because, for example, even very large elongation of one $\mathrm{C}-\mathrm{H}$ bond by $0.1 \AA$ leads to changes of the bond geminal amplitudes $u, v$, and $w$ not exceeding 0.003. The same applies to the averages of the pseudospin $(\hat{\tau})$ operators.

Linear Response of Hybridization to Bond Elongation. Let us first consider the relation between hybridization and elongation of the $\mathrm{C}-\mathrm{H}$ bond. For this end we need the mixed second-order derivatives coupling the bond stretching with the hybridization ESPs. For every $\mathrm{C}-\mathrm{H}$ bond in methane we can introduce a diatomic coordinate frame with the $\zeta$ axis directed along the bond and express the resonance integral as:

$$
\beta_{r_{m} l_{m}}^{C H_{m}}=\beta_{\sigma \sigma}^{C H} S_{m}+\beta_{\zeta \sigma}^{C H} v_{m \zeta},
$$

where the subscript $m$ enumerates the $\mathrm{C}-\mathrm{H}$ bonds. In the case of methane, $s_{m}=\frac{1}{2}$ and $v_{m \zeta}=\sqrt{3} / 2$. Changing the bond length causes the response of the vector $\delta \vec{\omega}_{l}$ to be exactly zero (vector product of collinear vectors) because the directions of the chemical bonds and the HOs coincide in the reference structure of methane. At the same time, the response of the shape of the hybridization tetrahedron represented by the vector $\delta \vec{\omega}_{b}$ is nonvanishing and can be written as: ${ }^{29}$

$$
\delta \vec{\omega}_{b}=-\frac{\sqrt{3}}{4} \cdot \frac{\sqrt{3} \theta_{\sigma \sigma}^{C H}-\theta_{\zeta \sigma}^{C H}}{\sqrt{3} \beta_{\sigma \sigma}^{C H}+\beta_{\zeta \sigma}^{C H}} \vec{e}_{m} \delta r_{m},
$$

where the derivative of $\beta_{\mu \nu}$ with respect to the interatomic distance is $\theta_{\mu \nu}, \vec{e}_{m}$ is the unit vector directed along the $C H_{m}$ bond and $\delta r_{m}$

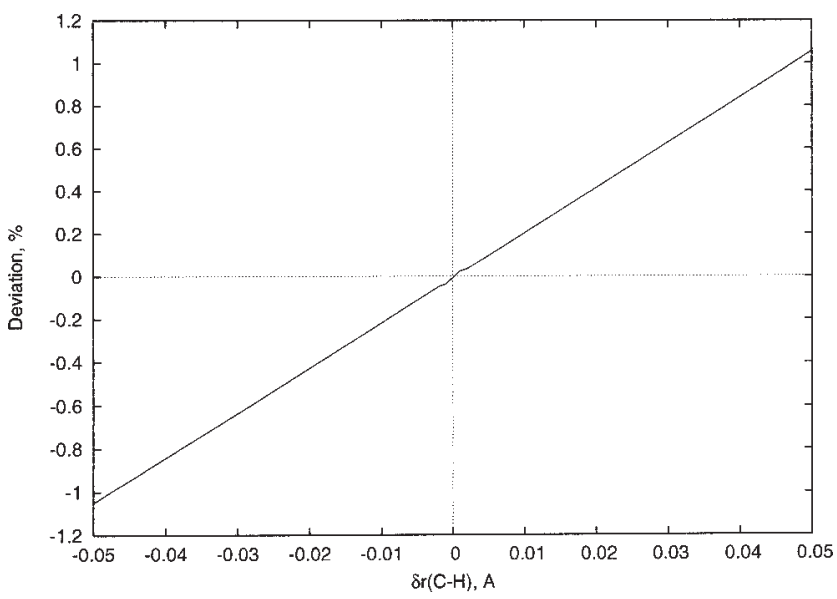

Figure 1. Deviation of the linear response estimate of the $\delta$ angle from the precise one for elongation of the $\mathrm{C}-\mathrm{H}$ bond in methane.

is the variation of the length of the same $\mathrm{C}-\mathrm{H}$ bond. The denominator in this expression corresponds to the eigenvalue of the $\nabla_{x} \nabla_{x} E$ matrix referring to $\vec{\omega}_{b}$, while the numerator corresponds to the block of the $\nabla_{x} \nabla_{q} E$ matrix where $q$ is the bond length $r_{m}$ and $x$ is $\vec{\omega}_{b}$.

Formula (37) gives the analytical expression for the coupling coefficient between the bond elongation (in $\AA$ ) multiplied by unit vector of this bond direction and changes of pseudorotation angles $\delta \vec{\omega}_{b}$ in methane (in radians). Its numerical value $C_{1}$ is $0.2764 \mathrm{rad}$. $\AA^{-1}$. This distortion corresponds to the following form for the matrix of small transformation of the whole set of HOs [matrix $H$ in eq. (30)]:

$$
\left(\begin{array}{cccc}
1 & -\delta & -\delta & -\delta \\
\delta & 1 & 0 & 0 \\
\delta & 0 & 1 & 0 \\
\delta & 0 & 0 & 1
\end{array}\right)
$$

where the linear response estimate for $\delta$ is $C_{1} \cdot(\delta r / \sqrt{3})$. This form of the transformation matrix is perfectly numerically reproduced both in the FA and TA pictures. Figure 1 represents the relative deviation of $\delta$ estimated in the linear response approximation with respect to the exact value obtained by the energy minimization (both values are obtained within the FA picture). This figure shows that the linear response is good even for large distortions (deviation from linearity is only about $1 \%$ for stretching of $0.05 \AA$ ). The approximately linear dependence of the error itself on the variation of the bond length certifies that the second-order estimate should perfectly describe the $\delta$ parameter obtained by the energy minimization.

Linear Response of Hybridization to Valence Angle Deformation. The linear response relations between the molecular shape and the shape of hybridization tetrahedron are rather tricky due to the complex structure of the hybridization manifold. The molecular shape can be formally characterized by unit vectors with the origin at an atom considered, taken as a center, and pointing to those 
bonded to the central one. In the case of methane, the deformations of thus defined coordination polyhedron are small rotations of unit vectors $\vec{e}_{m}$ directed from the carbon atom to the hydrogen atoms. Their small rotations $\delta \vec{\varphi}_{m}$ form an eight-dimensional space that decomposes to a direct sum of two subspaces: one three-dimensional corresponding to rotations of the molecule as a whole and another five-dimensional corresponding to independent variations of valence angles. The former one is precisely mapped on the three-dimensional space of quasirotations $\delta \vec{\omega}_{l}$ while the latter (five-dimensional) one is mapped on the three-dimensional space of pseudorotations $\delta \vec{\omega}_{b}$ corresponding to changes of the shape of the hybridization tetrahedron. ${ }^{29}$ Due to very general theorems of linear algebra, ${ }^{43}$ there exists at least a two-dimensional kernel in the space of deformations of a molecular shape that maps to zero deformation of hybridization tetrahedron. In ref. 29, the term "hybridization incompatible" has been coined for the deformations from this kernel. The structure of deformations laying in the kernel of the mapping is quite simple: they are produced by equal variations of opposite (spiro) valence angles. In contrast, the variations that correspond to increase of one valence angle by $\delta \chi$ and decrease of its spiro counterpart by the same value fall into "coimage" of this mapping, that is, to the subspace of one-to-one maps to the space of pseudorotations $\delta \vec{\omega}_{b}$. The deformations in the coimage can be called "hybridization compatible." It is clear that only these latter variations should be considered. It is also clear that any variation of the valence angle is a sum of equal amounts of hybridization compatible and hybridization-incompatible deformations. The denominator in the linear response relation [eq. (35)] is the same as for eq. (37) while the relevant block of the $\nabla_{x} \nabla_{q} E$ matrix (with $q$ taken as a difference of two opposite valence angles) is proportional to $\beta_{\zeta \sigma}^{\mathrm{CH}}$. Applying the linear response technique to the "hybridization-compatible" variation of two spiro valence angles we obtain:

$$
\delta \vec{\omega}_{b}=-\frac{\beta_{\zeta \sigma}^{C H}}{\sqrt{2}\left(\sqrt{3} \beta_{\sigma \sigma}^{C H}+\beta_{\zeta \sigma}^{C H}\right)} \delta \chi \vec{k},
$$

where $\vec{k}$ is the ort directed along the symmetry axis preserved during this distortion. ${ }^{29}$

The coupling coefficient between the change of the pseudorotation vector and totally hybridization-compatible deformation of valence angles can be easily found by eq. (39). Its numerical value $C_{2}$ is -0.20734 for the equilibrium interatomic distance. The considered distortion produces the following $\mathrm{HO}$ transformation matrix [matrix $H$ in eq. (30)]:

$$
\left(\begin{array}{cccc}
1 & 0 & 0 & -\delta \\
0 & 1 & 0 & 0 \\
0 & 0 & 1 & 0 \\
\delta & 0 & 0 & 1
\end{array}\right),
$$

where the linear response estimate for $\delta$ is $C_{2} \cdot \delta \chi$. The change of angle between HOs (i.e., between the vector parts $\vec{v}_{m}$ of the quaternions representing the HOs) is $-2 \sqrt{2} \delta / 3$ for small values of $\delta$. The above form of the HO transformation matrix is perfectly confirmed by our numerical calculations performed within the FA picture even for very large distortions, which is a consequence of

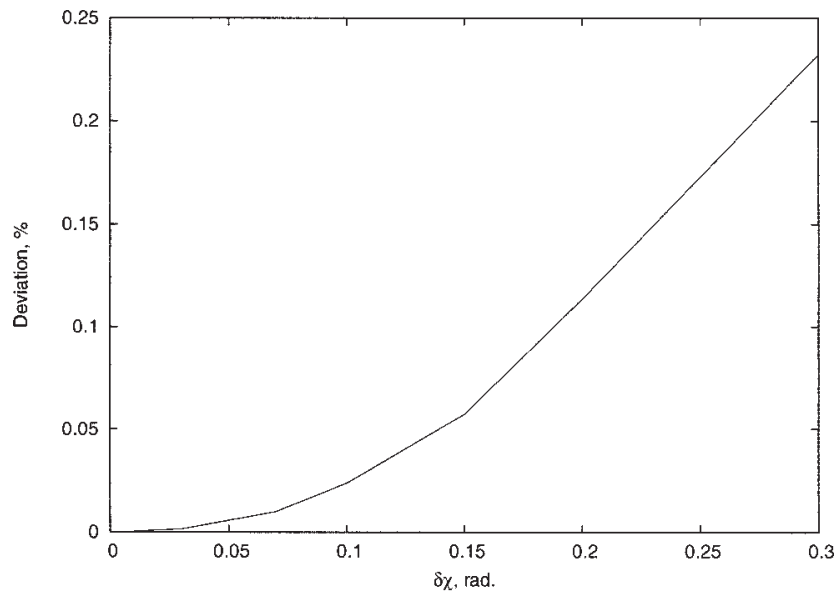

Figure 2. Deviation of the linear response estimate of the $\delta$ angle [eq. (40)] from the precise one for angular distortion of methane.

the mathematical structure of the hybridization manifold described above. Figure 2 represents the relative deviation between the linear response estimate of the $\delta$ parameter in eq. (40) and its value coming from the energy minimization procedure. These data show that the linear response estimate performs very well even for improbably large distortions [the deviation from the linear response estimate is smaller than $0.25 \%$ for the distortion of $0.3 \mathrm{rad}$ (about $\left.17^{\circ}\right)$ ].

The smallness of the coupling coefficient $C_{2}$ even for the totally hybridization-compatible deformations allows to qualitatively understand certain features of the electronic structure of cyclopropane as it appears in the SLG approach: a very large distortion of the $\mathrm{C}-\mathrm{C}-\mathrm{C}$ valence angle from the tetrahedral to $60^{\circ}$ one leads only to a relatively small distortion of the corresponding interhybrid angle. We model this process by strongly deforming the methane molecule. Simple estimate is based on eqs. (39) and (40) and runs as follows. The valence angle deformation when going from methane to cyclopropane is of $49.5^{\circ}\left(=109.5^{\circ}-60^{\circ}\right)$; only one-half of it is hybridization compatible; after multiplying by $C_{2}$ this yields the value of the interhybrid angle between the HOs corresponding to the "untouched" $\mathrm{C}-\mathrm{H}$ bonds of $114.5^{\circ}$ (i.e., the angle variation amounts only $5^{\circ}$ ). From the energy minimum condition for hydrides it follows that the $\mathrm{C}-\mathrm{H}$ bonds indeed must follow the directions of the HOs. Numerical experiments performed with the use of the SLG-MINDO/3 method show that if one of the $\mathrm{H}-\mathrm{C}-\mathrm{H}$ valence angles is fixed at the cyclopropane value of $60^{\circ}$ the energy minimum corresponds to its spiro counterpart of $115^{\circ}$. These results can be directly compared with the experimental $\mathrm{H}-\mathrm{C}-\mathrm{H}$ valence angle in the cyclopropane molecule, which equals $115.1^{\circ}$.

An analogous estimate can be applied to cyclobutane. In this case, we consider the distorted methane molecule with a $90^{\circ}$ angle. The response of the HOs to the deformation is proportional in our model to the deviation of the valence angle from the tetrahedral one. The deviation of the $\mathrm{C}-\mathrm{C}-\mathrm{C}$ angle from the tetrahedral one in cyclobutane $\left(19.5^{\circ}\right)$ amounts to $40 \%$ of that in cyclopropane. Therefore, we can expect that about the same ratio will be observed for the deviations of the $\mathrm{H}-\mathrm{C}-\mathrm{H}$ valence angle from the 
tetrahedral one in the cyclobutane and cyclopropane molecules. In fact, this ratio in the SLG-MINDO/3 numerical experiment is about $39 \%$

\section{Estimates of Parameters of the Standard MM Force Fields} Based on DMM

Announced transition from the DMM model of molecular PES to a model dependent on molecular geometry is formally obtained by inserting eq. (35) into eq. (34), which yields:

$$
\begin{aligned}
E=E_{0}+\frac{1}{2}( & \left.-q_{0}\left|\nabla_{q} \nabla_{q} E\right| q-q_{0}\right) \\
& -\frac{1}{2}\left(q-q_{0}\left|\nabla_{q} \nabla_{x} E\left(\nabla_{x} \nabla_{x} E\right)^{-1} \nabla_{x} \nabla_{q} E\right| q-q_{0}\right) .
\end{aligned}
$$

It apparently consists of two contributions: (1) the leading onethe second derivatives of the energy [eq. (32)] with respect to the geometry parameters $q$, and (2) a smaller correction appearing as a result of projecting out the ESPs related to the hybridization tetrahedra. Due to the SLG form of the wave function the energy expression [eq. (32)] is naturally represented as a sum of atom and bond increments. Staying for the sake of simplicity within the FA picture we can conclude that the only geometry-dependent contribution is that proportional to the resonance integrals of respective bonds. These contributions depend mainly on the natural nuclear coordinates: ${ }^{40}$ bond lengths and valence angles entering the definition of the standard MM force fields. This makes sensible to consider the geometry dependence of individual bond energies. For a pair of singly bonded atoms the corresponding energy is:

$$
E_{R_{m}}+E_{L_{m}}+E_{R_{m} L_{m}}^{\mathrm{bond}}+E_{R_{m} L_{m}}^{\mathrm{nonond}}
$$

which for a symmetric bond may be written as:

$$
\begin{aligned}
E=2\left(U_{m}-\gamma_{R_{m} L_{m}}\right)+\frac{g_{m}}{2}( & \left.1-\frac{1}{\Gamma\left(\zeta_{m}\right)}\right)+\frac{\gamma_{R_{m} L_{m}}}{2}\left(1+\frac{1}{\Gamma\left(\zeta_{m}\right)}\right) \\
& -2 \beta_{r_{m} L_{m}}^{R_{m} L_{m}} \frac{\zeta_{m}}{\Gamma\left(\zeta_{m}\right)}+Z_{R_{m}} Z_{L_{m}} D_{R_{m} L_{m}},
\end{aligned}
$$

where $U_{m}$ is the mean arithmetic value of the one-center electron core attraction parameters $U_{m}^{r}$ and $U_{m}^{l}$.

We consider in more detail the energy curve for the $\mathrm{C}-\mathrm{H}$ bond. The curve corresponding to the $s p^{3}$ hybridization of carbon atom and to the symmetric TA picture [eq. (20)] is given by Figure 3 . It has correct qualitative behavior for all interatomic separations. The minimum depth on this curve is approximately -0.23 a.u. and can be considered as the "pure" energy of the $\mathrm{C}-\mathrm{H}$ bond. It is generally accepted in the literature that the energy of the $\mathrm{C}-\mathrm{H}$ bond is approximately 0.15 a.u. At the same time the latter value is thermodynamic one, while our value is obtained by extracting the contributions to the energy intrinsic to this bond and excluding the interaction between the bonds. The difference between the thermodynamic value for the bond energy and that obtained from the SLG energy in the FA picture can be explicitly written in quite simple form:

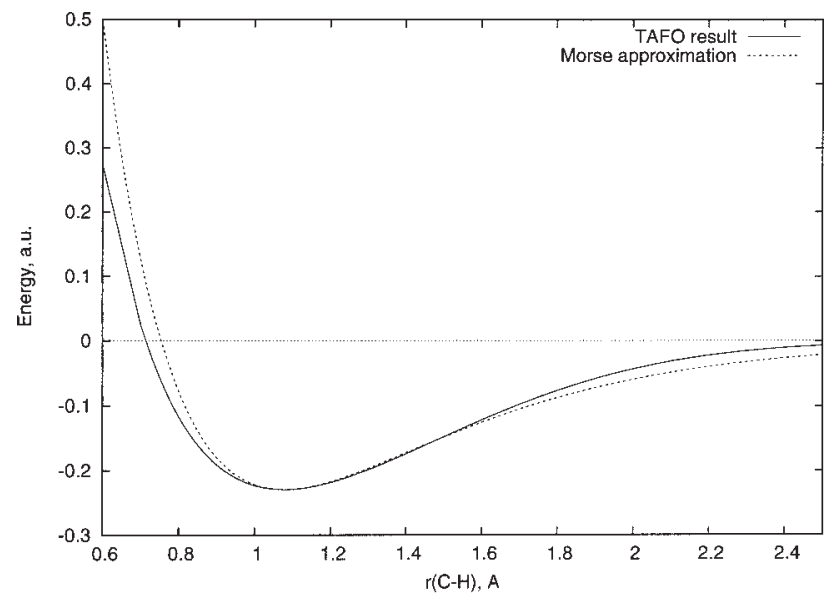

Figure 3. $\mathrm{C}-\mathrm{H}$ bond energy profile for the methane molecule obtained within the symmetric TAFO approximation.

$$
1 / 4\left[U_{s}(C)+3 U_{p}(C)+3 g_{t t^{\prime}}^{C}+6 D_{H H}-E_{A}(C)\right]
$$

where $E_{A}(C)$ is the energy of the nonhybridized carbon atom. Adding this value to the minimum of the energy curve for the $\mathrm{C}-\mathrm{H}$ bond gives the value close to the thermodynamic one because the heats of formation are well reproduced within the SLG-MINDO/3 method.

It is interesting to compare the form of the bond energy curve (Fig. 3) with the Morse potential. To this purpose we tried to approximate the curve of Figure 3 by the Morse function $D_{0}[1-$ $\left.\exp \left(-a\left(r-r_{e}\right) / r_{e}\right)\right]^{2}$ by minimizing the area between two curves in the interval from 0.72 to $2.50 \AA$. With the parameters $D_{0}$ and $r_{e}$ fixed at the values equal to the minimum depth and position on the curve $(0.2295$ a.u. and $1.078 \AA$ ) the optimal value of parameter $a$ is then 2.306, but with these parameters two curves are, in fact, quite different (the area between curves is almost $11 \%$ of area between the bond energy curve and the abscissa). If we optimize all three parameters of the Morse curve they become slightly modified $D_{0}=0.2333$ a.u., $r_{e}=1.045 \AA$, and $a=2.295$. This reduces the area between the curves by $30 \%$. It should be concluded that the energy profile in the TA approximation is not particularly well reproduced by whatever Morse curve.

To estimate the parameters of harmonic force fields we consider the symmetric correlated single bond, where the energy can be obtained without any reference to its environment. In our case, the derivative of the bond energy with respect to a geometry parameter $q$ has the form:

$$
\begin{aligned}
\frac{\partial E_{m}}{\partial q}=Z_{R_{m}} Z_{L_{m}} \frac{\partial D_{R_{m} L_{m}}}{\partial q}-2 \frac{\zeta_{m}}{\Gamma\left(\zeta_{m}\right)} & \frac{\partial \beta_{r_{m} l_{m}}^{R_{m} L_{m}}}{\partial q} \\
& -\frac{1}{2}\left(1-\frac{1}{\Gamma\left(\zeta_{m}\right)}\right) \frac{\partial \gamma_{R_{m} L_{m}}}{\partial q},
\end{aligned}
$$

where the derivatives of different ESPs with respect to geometry variables exactly cancel each other so that the final expression for the energy derivative acquires the form expected from the pertur- 
Table 2. DMM-Based Estimates of the MM Force Field Parameters to Those Accepted in Some Standard MM Parameterizations.

\begin{tabular}{lcc}
\hline$r_{0}^{\mathrm{CH}} \AA$ & $k_{\mathrm{CH}} \mathrm{mdyn} / \AA$ & $k_{\mathrm{HCH}} \mathrm{mdyn} / \mathrm{deg}$ \\
\hline FA: 1.069 & 8.30 & 0.509 \\
$\mathrm{TA}_{\text {symm }}: 1.078$ & 7.77 & \\
TA $_{\text {pert }}: 1.096$ & 7.17 & \\
& Standard MM: & [39]: 0.549 \\
[2]: 1.113 & {$[2]: 4.5 \div 4.7$} & {$[41]: 0.508$} \\
[39]: 1.105 & {$[40]: 5.31$} & {$[40]: 0.493$} \\
[41]: 1.090 & {$[42]: 7.90$} & \\
\hline
\end{tabular}

bative analysis of the ESPs given above. If $q$ is the interatomic distance, setting the derivative to zero yields the equation for determining the minimum position. Results are given in Table 2. In the limit $\zeta_{m} \gg 1$ we recover the equilibrium geometry condition for the FA picture. The meaning of other notation in the Table 2 is following. The $\mathrm{TA}_{\text {symm }}$ refers to a TA estimate for the symmetric bond (bond asymmetry-polarity-terms omitted), while $\mathrm{TA}_{\text {pert }}$ refers to the perturbative inclusion of bond asymmetry effects to the TA picture with use of the $\mu_{0}$ parameter. All estimates are quite reasonable. At the same time, the latter one looks more promising because it perfectly corresponds to the equilibrium interatomic separation in methane and other hydrocarbons.

The same concepts can be used to determine the elasticity constant for the bond stretching by taking the second derivative of the energy with respect to the bond length. In the FA picture we get:

$$
k_{R_{m} L_{m}}=\left(Z_{R_{m}} Z_{L_{m}} \frac{d^{2} D_{R_{m} L_{m}}}{d r_{R_{m} L_{m}}^{2}}-2 \frac{\partial^{2} \beta_{r_{m} l_{m}}^{R_{m} L_{m}}}{\partial r_{R_{m} L_{m}}^{2}}-\frac{1}{2} \frac{d^{2} \gamma_{R_{m} L_{m}}}{d r_{R_{m} L_{m}}^{2}}\right)_{r_{r_{L} L_{m}}^{0}} .
$$

We see from Table 2 that at least for one of the "experimental" estimates for the stiffness of the $\mathrm{C}-\mathrm{H}$ bond ${ }^{42}$ the agreement is quite acceptable. The deviation from other cited values may be understood because the bond stretch parameters fitted by other authors in the context of the structure-oriented MM schemes are implicitly loaded with the average effects of surrounding atoms. This disagreement cannot be ascribed to the effects of the projected out Jacobi angles. Indeed, the off-diagonal constant coupling stretching for two incident $\mathrm{C}-\mathrm{H}$ bonds in the methane molecule can be written as:

$$
K_{\text {off }}=\frac{1}{4 \sqrt{3}} \frac{\left(\sqrt{3} \theta_{\sigma \sigma}^{C H}-\theta_{\zeta \sigma}^{C H}\right)^{2}}{\sqrt{3} \beta_{\sigma \sigma}^{C H}+\beta_{\zeta \sigma}^{C H}}
$$

The only reason why this term appears is the deformation of the carbon hybridization tetrahedron [eq. (37)] effectively coupling stretchings of two $\mathrm{C}-\mathrm{H}$ bonds. Its estimated magnitude is only $0.120 \mathrm{mdyn} / \AA$, which is in an agreement with its IR estimate 0.03 mdyn $/ \AA^{40}$ to the order of magnitude. This estimate, however, establishes the scale of the corresponding effects. One can see that the DMM specific corrections to the bare estimates of the harmonic stretching constants [eq. (46)] are small. Nevertheless, in the cases when the bare harmonic constant vanishes (like the considered off-diagonal constant does) the DMM correction gives an estimate correct to the order of magnitude and allows solving the question on the presence of the off-diagonal terms on a purely theoretical basis.

Analogous treatment of the energy terms quadratic in valence angles' deformations yields the bare estimate for the harmonic bending constant in the form:

$$
k_{H C H}=\frac{\sqrt{3}}{2} \beta_{\zeta \sigma}^{C H},
$$

From Table 2 one can see that the elasticity constants for bending force fields are in a good agreement with the values accepted in the literature.

\section{Discussion}

In the previous section we provided the exclusion of the angular variables characterizing the shapes and orientations of the hybridization tetrahedra from the mechanistic DMM model of molecular PES. This results in a model announced in the Introduction, which is similar to the standard MM models, but is obtained by the sequential derivation from the QM (SLG) model of molecular electronic structure. As is mentioned, the transferability of the ESPs characterizing chemical bonds in molecules and linear response relations for hybridization ESPs are main components of deriving the MM theory of molecular PESs from the corresponding QM theory. Both these features have been mathematically derived and numerically checked in Section 3.

From a quantitative point of view, the concept of transferability have gotten some attention in two related areas. First, we mention the estimates of that of the semiempirical quantum chemical parameters given in ref. 44. Analogously in ref. 45 the problem of constructing transferable dynamical matrices in relation to analysis of vibrational spectra has been considered. The importance of the transferability of the geminals has been pointed out, yet in ref. 24 as a prerequisite for that of the bond energy. However, in ref. 24 the geminal transferability had not been proven, and the authors tried to prove directly that of the MM bond stretching force fields. The latter is an important characteristic of the MM approach itself. Here, we present a step towards quantitative analysis of the transferability and eventually to the mathematical proof of the later for the MM force fields by proving the transferability of the density matrix elements - equivalent to that of the geminal amplitudes, but more directly related to the energy. Under the assumptions given by eqs. (15) and (16), the averages of the pseudospin operators (and, thus, all the bond ESPs) are invariant in that sense that they do not depend on the environment of the bond under consideration and even on the nature and the hybridization of the atoms the bond connects. This corresponds to the FA picture (see above and ref. 28). It is important that the invariance (at the established level of precision) of the density matrix elements can be proven only for the basis of the variationally determined HOs-a specific characteristic of the SLG approach. ${ }^{25,26}$ In the basis of AOs, the density matrix elements are not invariant even 
approximately. Although the SCF approximation formally suffice to obtain these invariant results it is very crude, which allows to pose further question: to what extent the density ESPs' invariance may stand further improvements of the description and whether it is possible to relate the invariance of the density matrix elements with the transferability of the MM force fields. To answer these questions we notice that the invariant values of ESPs can be improved by perturbative corrections (the TA picture) reflecting all diversity of chemical compositions and environments the bond may occur in. All the variety of perturbations is characterized by two small dimensionless parameters: $\zeta_{m}^{-1}$ [eq. (21)] and $\mu_{m}$ [eq. (23)]. Both parameters depend on the atoms connected by the bond, their separation, and their hybridization. The perturbative treatment allows estimation of the precision of transferability. For example, using eqs. (22), (24), and (25), we conclude that the bond order is transferable up to a second order with respect to both $\zeta_{m}^{-1}$ and $\mu_{m}$; the ionicity (the total weight of the ionic configurations) is transferable up to a second order with respect to $\mu_{m}$ and up to a first order with respect to $\zeta_{m}^{-1}$; the bond polarity is transferable up to a first order with respect to both $\zeta_{m}^{-1}$ and $\mu_{m}$. The second order transferability of bond orders explains to a certain extent the success of the concept of "single bond" suitable for a large variety of chemical bonds. Within this picture all specific characteristics of the force fields are loaded into parameters of the (effective) Hamiltonian, which are numbers specific either for a given atom in certain hybridization state or for a pair of such hybridization states of atoms - ends of the bond. Although the density ESPs can be considered as constants independent of any details of molecular composition or geometry, the force fields that are basically sums of products of ESPs by matrix elements of the molecular Hamiltonian are geometry dependent and composition specific. The force fields thus obtained are expected to be the same for the same composition of the bond and to depend weakly (to the extent of the variance of the $\mu_{m 1}$ parameters) on the environment. These properties are basically much more than necessary for substantiating the MM-like description.

\section{Conclusions}

In the present article we discussed the problem of deriving the MM representation of the molecular PES from a relevant QM description. Using the SLG wave function we analyzed the ESPs related to bond geminals and to hybridization tetrahedra. It was shown that the bond-related parameters can be represented as functions of parameters of the MINDO/3 Hamiltonian in the HO basis, transferable from one molecule to another. The functional form of the ESPs found is valid at arbitrary interatomic separations. At the interatomic separations close to the equilibrium bond lengths characteristic for the MM-like treatments, two approximations, both suitable for substantiation of the ESPs transferability, were considered. One is the fixed geminal amplitudes approximation, which results in perfectly transferable numbers, referring to the ESPs in question. Another, more precise, is the tuned geminal amplitudes approximation, which takes into account small corrections to the invariant ESPs. Two small parameters characterizing specificity of the bond and effects of its environment were introduced. By this the whole manifold of quantum chemical parameters defining the effective bond Hamiltonian boils down to only two relevant parameters, $\zeta_{m}^{-1}$ and $\mu_{m}$. The presence of such, only two-dimensional manifold and smallness of the parameters, for a wide range of bonds in quite different environments essentially explains the transferability of the density-related ESPs. This allows for a family of mechanistic models describing molecular PESs in terms of hybridization tetrahedra with interactions dependent on distances between their centers and on mutual orientations. Linear response relations for variation of hybridization parameters due to elongation of chemical bonds or specific changes of valence angles are considered. They allow exclusion of the angular variables describing the shapes and orientations of hybridization tetrahedra, and to represent the molecular energy in both the FA and TA approximations as that of the system of interacting point masses ${ }^{46}$ ("balls and springs" picture), depending on the molecular geometry only. This energy has a form of a sum of local (bond) increments corresponding to the force fields of the standard MM. The estimates of the parameters of these force fields coming from the analytical expressions are compared with those obtained in numerical experiments showing the high accuracy of analytical estimates. The reasons for this possibility are the transferability of the bond-related ESPs and the linear response relations for the hybridization tetrahedra. They both are numerically tested for their precision.

The results obtained provide both the theoretical proof and numerical support to the constructs of the QM/MM junction given in refs. 8 and 30. Although some direct interest to using semiempirical methods as QM components of the hybrid QM/MM methods is reported in the literature, ${ }^{47-50}$ the main goal reached by the present work is to develop a general theoretical framework potentially applicable to a more refined QM method and to provide at least the most simple numerical implementation for this framework. The feasibility of the proposed approach for the nonempirical techniques is highly probably justified by analysis given in ref. 51, which shows the existence of an "effective" minimal basis (actually of the core and valence type) of one-electron stateseffective hybrid orbitals as extracted from the Hartree-Fock calculations in the DZP or even TZP basis-and practically accounting for the whole electronic structure of the molecule at hand. Also, the success of the schemes permitting to perform decomposition of the total energy into local (atomic and diatomic) contributions is to be mentioned in this connection. ${ }^{52,53}$ This gives hope that the criteria for the QM method possibly underlying the MMlike description of the molecular PES can also be satisfied by certain $a b$ initio methods.

\section{Acknowledgments}

This work was completed during the stay of A.M.T. in the RWTH Aachen in the frame of the Alexander von Humboldt Postdoctoral Fellowship, which is gratefully acknowledged, as is the kind hospitality of Prof. R. Dronskowski. The authors are grateful to the referees for valuable comments. A.L.T. gratefully acknowledges valuable discussions with Profs. I. A. Misurkin and N. F. Stepanov. Prof. I. Mayer read the final version of the present article and gave valuable comments and suggestions, which is gratefully acknowledged. 


\section{References}

1. Dashevskii, V. G. Conformations of Organic Molecules; Khimiya: Moscow, Russia, 1974.

2. Burkert, U.; Allinger, N. Molecular Mechanics; ACS: Washington, DC, 1982.

3. Goedecker, S. Rev Mod Phys 1999, 71, 1085.

4. Wu, S. Y.; Jayanthi, C. S. Phys Rep 2002, 358, 1.

5. Warshel, A.; Levitt, M. J Mol Biol 1976, 103, 227.

6. Gao, J. In Reviews in Computational Chemistry; VCH Publishers, Inc.: New York, 1996.

7. Sherwood, P. In Modern Methods and Algorithms of Quantum Chemistry; Grotendorst, J., Ed.; NIC Series, 3, John von Neumann Institute for Computing: Jülich, 2000.

8. Tchougréeff, A. L.; Tokmachev, A. M. In Advanced Topics in Theoretical Chemical Physics; Maruani, J.; Lefebvre, R.; Brändas, E., Eds.; Kluwer: Dordrecht, 2003.

9. Tchougréeff, A. L. Phys Chem Chem Phys 1999, 1, 1051.

10. Tokmachev, A. M.; Tchougréeff, A. L.; Misurkin, I. A. J Mol Struct (Theochem) 2000, 506, 17.

11. Tokmachev, A. M.; Tchougréeff, A. L. Int J Quantum Chem 2001, 84, 39

12. Allinger, N. L. J Am Chem Soc 1977, 99, 8127.

13. Allinger, N. L.; Yuh, Y. H.; Lii, J.-H. J Am Chem Soc 1989, 111, 8551.

14. Jorgensen, W. L.; Tirado-Rives, J. J Am Chem Soc 1988, 110, 1657.

15. Brooks, B. R.; Bruccoleri, R. E.; Olafson, B. D.; States, D. J.; Swaminathan, S.; Karplus, M. J Comp Chem 1983, 4, 187.

16. Cornell, W. D.; Ciepak, P.; Bayly, C. I.; Gould, I. R.; Merz, K.; Ferguson, D. M.; Spellmeyer, D. C.; Fox, T.; Caldwell, J. W.; Kollman, P. J Am Chem Soc 1995, 117, 5179.

17. Halgren, T. A. J Comp Chem 1996, 17, 490.

18. Allinger, N. L.; Chen, K.; Lii, J.-H. J Comp Chem 1996, 17, 642.

19. Comba, P.; Hambley, T. W. Molecular Modeling; VCH Publishers: Weinheim, 1995.

20. Kozelka, J. In Metal Ions in Biological Systems, Sigel, A.; Sigel, H., Eds.; Marcel Dekker Inc.: New York, 1996, vol. 33

21. Pletnev, I. V.; Melnikov, V. L. Russ Chem Bull 1997, 46, 1278.

22. Daudey, J.-P.; Malrieu, J.-P.; Rojas, O. In Localization and Delocalization in Quantum Chemistry, Vol. 1. Atoms and Molecules in the Ground State; Chalvet, O.; Daudel, R.; Diner, S.; Malrieu, J.-P., Eds.; D. Reidel Publ.: Dordrecht, 1975.

23. Diner, S.; Malrieu, J.-P.; Claverie, P. Theor Chim Acta 1969, 13, 1.

24. Allen, T. L.; Shull, H. J Chem Phys 1961, 35, 1644.

25. Tokmachev, A. M.; Tchougréeff, A. L. J Comp Chem 2001, 22, 752.

26. Tokmachev, A. M.; Tchougréeff, A. L. J Phys Chem A 2003, 107, 358.
27. Tokmachev, A. M.; Tchougréeff, A. L. Int J Quantum Chem 2001, 85, 109.

28. Tokmachev, A. M.; Tchougréeff, A. L. Int J Quantum Chem 2002, 88, 403.

29. Tchougréeff, A. L.; Tokmachev, A. M. Int J Quantum Chem 2004, 96, 175.

30. Tchougréeff, A. L.; Tokmachev, A. M. Int J Quantum Chem 2004, $100,667$.

31. Tchougréeff, A. L. J Mol Struct (Theochem) 2003, 630, 243.

32. Fock, V. A.; Veselov, M. G.; Petrashen, M. I. J Exp Theor Phys 1940, $10,723$.

33. Weinbaum, S. J Chem Phys 1933, 1, 593.

34. Bingham, R. C.; Dewar, M. J. S.; Lo, D. H. J Am Chem Soc 1975, 97, 1302.

35. Parks, J. M.; Parr, R. G. J Chem Phys 1958, 28, 335.

36. Abrikosov, A. A.; Gor'kov, L. P.; Dzyaloshinskii, I. Ye. Quantum Field Theoretical Methods in Statistical Physics; Pergamon: Oxford, 1965

37. Poincaré, H. La Science et l'Hypotése; Flammarion: Paris, France, 1923.

38. Pupyshev, V. I.; Panchenko, Y. N.; Bock, C. W.; Pongor, G. J Chem Phys 1991, 94, 1247.

39. Ermer, O.; Lifson, S. J Am Chem Soc 1973, 95, 4121.

40. Volkenstein, M. V.; Gribov, L. A.; Elyashevich, M. A.; Stepanov, B. I. Molecular Vibrations; Nauka: Moscow, Russia, 1972.

41. Chang, S.; McNally, D.; Shary-Tehrany, S.; Hickey, M. J.; Boyd, R. H. J Am Chem Soc 1970, 92, 3109.

42. Pearson, R. G. Symmetry Rules for Chemical Reactions; Wiley: New York, 1976.

43. Kostrikin, A. I.; Manin, Yu. I. Linear Algebra and Geometry; Nauka: Moscow, Russia, 1986.

44. Fisher-Hjalmars, I. In Modern Quantum Chemistry. Istanbul Lectures; Sinanoğlu, O., Ed.; Academic Press: New York, 1965.

45. Stepanov, N. F.; Koptev, G. S.; Pupyshev, V. I.; Panchenko, Yu. N. In Modern Problems of Physical Chemistry; MSU Publ.: Moscow, Russia, 1979, vol. 11.

46. Meyer, A. Y. In Theoretical Models of Chemical Bonding, Part 1; Maksić, Z. B., Ed.; Springer: Heidelberg, 1989.

47. Ferenczy, G. G.; Csonka, G. I.; Náray-Szabó, G.; Ángyán, J. G. J Comp Chem 1998, 19, 38.

48. Náray-Szabó, G. Comput Chem 2000, 24, 287.

49. Reuter, N.; Dejaegere, A.; Maigret, B.; Karplus, M. J Phys Chem A 2000, 104, 1720

50. Field, M. J.; Albe, M.; Bret, C.; Proust-de Martin, F.; Thomas, A. J Comput Chem 2000, 21, 1088.

51. Mayer, I. J Phys Chem 1996, 100, 6249.

52. Mayer, I. Chem Phys Lett 2000, 332, 381 .

53. Mayer, I. Chem Phys Lett 2003, 382, 265 